\title{
A combinatorial proof of the non-vanishing of Hankel determinants of the Thue-Morse sequence
}

\author{
Yann Bugeaud Guo-Niu Han \\ Institut de Recherche Mathématique Avancée \\ Université de Strasbourg et CNRS \\ Strasbourg, France \\ \{bugeaud, guoniu\}@math.unistra.fr
}

Submitted: Oct 21, 2013; Accepted: Aug 7, 2014; Published: Aug 21, 2014

Mathematics Subject Classifications: 05A05, 11J82

\begin{abstract}
In 1998, Allouche, Peyrière, Wen and Wen established that the Hankel determinants associated with the Thue-Morse sequence on $\{-1,1\}$ are always nonzero. Their proof depends on a set of sixteen recurrence relations. We present an alternative, purely combinatorial proof of the same result. We also re-prove a recent result of Coons on the non-vanishing of the Hankel determinants associated to two other classical integer sequences.
\end{abstract}

Keywords: Hankel determinant; combinatorial proof; Thue-Morse sequence

\section{Introduction}

Let $\mathcal{C}(z)$ be a power series in one variable with rational coefficients,

$$
\mathcal{C}(z)=\sum_{k \geqslant 0} c_{k} z^{k}, \quad c_{k} \in \mathbb{Q}
$$

For $k \geqslant 1$ and $p \geqslant 0$, let

$$
H_{k}^{p}(\mathcal{C}):=\left|\begin{array}{cccc}
c_{p} & c_{p+1} & \ldots & c_{p+k-1} \\
c_{p+1} & c_{p+2} & \ldots & c_{p+k} \\
\vdots & \vdots & \ddots & \vdots \\
c_{p+k-1} & c_{p+k} & \ldots & c_{p+2 k-2}
\end{array}\right|
$$


be the $(p, k)$-order Hankel determinant associated to $\mathcal{C}(z)$. For simplicity, we write $H_{k}(\mathcal{C})$ instead of $H_{k}^{0}(\mathcal{C})$. The study of the non-vanishing of $(p, k)$-order Hankel determinants is an interesting question on its own, and this is the purpose of the present paper for the sequence $\left(c_{k}\right)_{k \geqslant 0}$ being the Thue-Morse sequence. However, we start the introduction by pointing out a motivation coming from Diophantine approximation and concerning the study of rational approximation to the real numbers $\mathcal{C}(1 / b)$, where $b \geqslant 2$ is an integer.

Let $\xi$ be an irrational, real number. The irrationality exponent $\mu(\xi)$ of $\xi$ is the supremum of the real numbers $\mu$ such that the inequality

$$
\left|\xi-\frac{p}{q}\right|<\frac{1}{q^{\mu}}
$$

has infinitely many solutions in rational numbers $p / q$. It follows from the theory of continued fractions that $\mu(\xi)$ is always greater than or equal to 2 , and an easy covering argument shows that $\mu(\xi)$ is equal to 2 for almost all real numbers $\xi$ (with respect to the Lebesgue measure). Furthermore, Roth's theorem asserts that the irrationality exponent of every algebraic irrational number is equal to 2 . The reader is directed to the monograph [5] for proofs and refinements of these assertions. It is in general a very difficult problem to determine the irrationality exponent of a given transcendental real number $\xi$, unless $\xi$ is given by its continued fraction expansion. Apart from more or less ad hoc constructions, there are only very few examples of transcendental numbers $\xi$ whose irrationality exponent is known. When they can be applied, the current techniques allow us most often only to get an upper bound for $\mu(\xi)$.

Recently, Bugeaud [2] developed a method for computing $\mu(\xi)$ when $\xi$ is a ThueMorse-Mahler number. Let

$$
\mathbf{t}=t_{0} t_{1} t_{2} \ldots=1-1-11-111-1-111-1 \ldots
$$

denote the Thue-Morse word on $\{-1,1\}$ defined by $t_{0}=1, t_{2 k}=t_{k}$ and $t_{2 k+1}=-t_{k}$ for $k \geqslant 0$. Alternatively, $t_{k}=1$ (resp. -1 ) if the number of 1's in the binary expansion of $k$ is even (resp. is odd). Let

$$
\mathcal{T}(z):=\sum_{k \geqslant 0} t_{k} z^{k}
$$

be the generating function of $\left(t_{k}\right)_{k \geqslant 0}$. It is proved in [2] that, for every integer $b \geqslant 2$, the irrationality exponent of the real number

$$
\mathcal{T}(1 / b)=\sum_{k \geqslant 0} \frac{t_{k}}{b^{k}}=1-\frac{1}{b}-\frac{1}{b^{2}}+\frac{1}{b^{3}}-\frac{1}{b^{4}}+\frac{1}{b^{5}}+\ldots
$$

is equal to 2. There are two main ingredients in the proof.

A first one is the fact that $\mathcal{T}(z)$ satisfies a functional equation, namely

$$
\mathcal{T}(z)=(1-z) \mathcal{T}\left(z^{2}\right)=\prod_{n \geqslant 0}\left(1-z^{2^{n}}\right),
$$


a key tool in Mahler's proof [4] that $\mathcal{T}(1 / b)$ is transcendental.

A second one is the non-vanishing of Hankel determinants associated with $\mathbf{t}$, a result established by Allouche, Peyrière, Wen and Wen [1].

Theorem (APWW). For every positive integer $k$, the Hankel determinant $H_{k}(\mathcal{T})$ is nonzero.

The proof given in [1] is long and difficult. It depends on a set of sixteen recurrence relations involving the $(p, k)$-order Hankel determinants and gives additional results on the values of the Hankel determinants $H_{k}^{p}(\mathcal{T})$.

Subsequently, Coons [3] considered the functions

$$
\mathcal{F}(z):=\sum_{k \geqslant 1} f_{k} z^{k}=\sum_{n=0}^{\infty} \frac{z^{2^{n}}}{1+z^{2^{n}}} \quad \text { and } \quad \mathcal{G}(z):=\sum_{k \geqslant 1} g_{k} z^{k}=\sum_{n=0}^{\infty} \frac{z^{2^{n}}}{1-z^{2^{n}}} .
$$

He proved that, for every integer $b \geqslant 2$, the irrationality exponent of $\mathcal{F}(1 / b)$ and $\mathcal{G}(1 / b)$ is equal to 2. To this end, he followed the method of [2], replacing the use of Theorem APWW by that of the next result (Theorem 2 of [3]).

Theorem (C). For every positive integer $k$, the Hankel determinants $H_{k}^{1}(\mathcal{F})$ and $H_{k}^{1}(\mathcal{G})$ are nonzero.

Coons' proof of Theorem $\mathrm{C}$ is of the same level of difficulty as the one of Theorem APWW. It is long and hard to follow.

The aim of this note is to provide a unified, combinatorial proof of both Theorems APWW and C. We believe that our approach is much simpler than that of $[1,3]$.

Our paper is organized as follows. The key combinatorial result, namely Theorem $\mathrm{J}$, is stated in Section 2, along with three equivalent lemmas. Complete proofs of these lemmas and theorem are given in Section 3. We gather in Section 4 some additional statements, which follow from our approach. Then, in Section 5, we show how Theorems APWW and $\mathrm{C}$ can be easily derived from Theorem J.

When nothing else is specified, the notation $a \equiv b$ means that the integers $a$ and $b$ are congruent modulo 2 .

\section{Permutations and involutions}

Throughout this text, $N$ denotes the set of non-negative integers. We introduce the sets

$$
J=\left\{(2 n+1) 2^{2 k}-1 \mid n, k \in N\right\}=\{0,2,3,4,6,8,10,11, \ldots\},
$$

and

$$
K=N \backslash J=\{1,5,7,9,13,17, \ldots\}=\left\{(2 n+1) 2^{2 k+1}-1 \mid n, k \in N\right\} .
$$

Let $\mathfrak{S}_{m}=\mathfrak{S}_{\{0,1, \ldots, m-1\}}$ be the set of all permutations on $\{0,1, \ldots, m-1\}$. In this section we prove the following result. 
Theorem $(\mathrm{J})$. (J1) For every integer $m \geqslant 1$, the number of permutations $\sigma \in \mathfrak{S}_{m}$ such that $i+\sigma(i) \in J$ for $i=0,1, \ldots, m-1$, is an odd number.

(J2) For every integer $m \geqslant 1$, the number of permutations $\sigma \in \mathfrak{S}_{m}$ such that $i+\sigma(i) \in$ $J$ for $i=0,1, \ldots, m-2$ (no constraint on $m-1+\sigma(m-1) \in N)$ is an odd number.

The proof is based on some combinatorial techniques. Since we want to enumerate permutations modulo 2, we can delete suitable pairs of permutations and the result will not be changed. The problem is then how to associate a given permutation with another to form a pair. Two methods are used in the present paper:

(1) taking the inverse $\sigma^{-1}$ of a given permutation $\sigma$;

(2) exchanging two values by letting $\sigma(i):=\sigma(j)$ and $\sigma(j):=\sigma(i)$.

Those two methods are fully described in the proof of Theorem $(J 1)$.

Throughout this paper we use three representations for permutations: the one-line, two-lines and the product of disjoint cycles. For example, we write

$$
\sigma \in \mathfrak{S}_{9}=516280374=\left(\begin{array}{l}
012345678 \\
516280374
\end{array}\right)=(0,5)(1)(2,6,3)(4,8)(7) .
$$

We choose to separate the elements of a cycle by commas.

Sometimes we write a list of sets under the two-line representations. If the set $A$ is under the index $i$, this means that $i+\sigma(i) \in A$. For example, the permutations in $(J 1)$ and $(J 2)$ are

$$
\left(\begin{array}{ccccccccc}
0 & 1 & 2 & 3 & 4 & 5 & 6 & 7 & 8 \\
\cdot & \cdot & \cdot & \cdot & \cdot & \cdot & \cdot & \cdot & \cdot \\
J & J & J & J & J & J & J & J & J
\end{array}\right)
$$

and

$$
\left(\begin{array}{ccccccccc}
0 & 1 & 2 & 3 & 4 & 5 & 6 & 7 & 8 \\
\cdot & \cdot & \cdot & \cdot & \cdot & \cdot & \cdot & \cdot & \cdot \\
J & J & J & J & J & J & J & J & N
\end{array}\right)
$$

respectively.

An involution is a permutation $\sigma$ such that $\sigma=\sigma^{-1}$. In the cycle representation of an involution every cycle is either a fixed point $(b)$ or a transposition $(c, d)$. For every set $A$, a transposition $(c, d)$ is said to be an $A$-transposition if $c+d \in A$ and $c+d$ is odd. This means also that there is an even number and an odd number in every $A$-transposition. Generally the order of the two numbers in a transposition does not matter. However, throughout this paper, we always write the even number before the odd number in every $A$-transposition.

For finite sets of positive integers $A, B$ and a non-negative integer $f$, let $\nu(A, f, B)$ be the number of involutions in $\mathfrak{S}_{A}$ such that all transpositions are $B$-transpositions and have exactly $f$ fixed points. Also let $\nu(A, f / g, B)=\nu(A, f, B)+\nu(A, g, B)$. For an infinite set $A$ let $\left.A\right|_{m}$ be the set composed of the $m$ smallest integers in $A$.

We state below three equivalent lemmas. The first (resp. second, third) assertion of any of these is equivalent to the first (resp. second, third) assertion of any of the other two lemmas. 
Lemma $(\mathrm{N})$. For $m \geqslant 1$ we have

$$
\begin{aligned}
\nu\left(\left.N\right|_{m}, 0 / 1, K\right) & \equiv 1 \\
\nu\left(\left.N\right|_{2 m-1}, 1, J\right) & \equiv 1 \\
\nu\left(\left.N\right|_{2 m}, 0 / 2, J\right) & \equiv 1
\end{aligned}
$$

Let

$$
P=\{0,3,4,7,8,11, \ldots\}=\{k \mid k=0,3 \quad(\bmod 4)\}
$$

and

$$
Q=\{1,2,5,6,9,10, \ldots\}=\{k \mid k=1,2 \quad(\bmod 4)\} .
$$

We define three transformations:

$$
\begin{array}{ll}
\beta: N \rightarrow N ; & k \mapsto \begin{cases}k / 2 & \text { if } k \text { is even } \\
(k-1) / 2 & \text { if } k \text { is odd }\end{cases} \\
\gamma: P \rightarrow N ; & k \mapsto \begin{cases}k / 4 & \text { if } k \text { is even } \\
(k-3) / 4 & \text { if } k \text { is odd }\end{cases} \\
\delta: N \rightarrow N ; & k \mapsto \begin{cases}k+1 & \text { if } k \text { is even } \\
k-1 & \text { if } k \text { is odd }\end{cases}
\end{array}
$$

The transformation $\beta$ is extended to the involutions $\sigma$ on $\left.N\right|_{m}$ such that all transpositions are $K$-transpositions, by applying $\beta$ on every number in the cycle representation of $\sigma$. The transformation $\beta$ for involutions is reversible, even though $\beta$ on $N$ is not reversible. For example

$$
\beta((7)(0,5)(6,3)(2)(8,1)(4))=(3)(0,2)(3,1)(1)(4,0)(2) .
$$

We do not know a priori whether the fixed point 3 is obtained from 6 or from 7 . We must look at the transposition $(3,1)$ first. It is obtained from the permutation $(6,3)$ since we know that an even number is always before an odd number in the transposition. Thus, we can recover the $K$-transpositions $(6,3)(0,5)(8,1)$. All the other numbers are fixed points, so these are $(7)(2)(4)$. If $(c, d)$ is a $K$-transposition, then

$$
\beta(c)+\beta(d)=\frac{c+d-1}{2}=\frac{(2 n+1) 2^{2 k+1}-1-1}{2}=(2 n+1) 2^{2 k}-1 \in J .
$$

In the same way, the transformation $\gamma$ is extended to the involutions $\sigma$ on $\left.P\right|_{m}$ such that all transpositions are $J$-transpositions, by applying $\gamma$ on every number in the cycle representation of $\sigma$. Again, the transformation $\gamma$ for involutions is reversible, even though $\gamma$ on $\left.P\right|_{m}$ is not reversible. For example

$$
\gamma((15)(0,11)(12,7)(4)(16,3)(8))=(3)(0,2)(3,1)(1)(4,0)(2) \text {. }
$$


If $(c, d)$ is a $J$-transposition and $c, d \in P$, then

$$
\gamma(c)+\gamma(d)=\frac{c+d-3}{4}=\frac{(2 n+1) 2^{2 k}-1-3}{4}=(2 n+1) 2^{2 k-2}-1 \in J .
$$

In fact we can check that the image sets of $\beta$ and $\gamma$ are identical, thus the transformation $\gamma^{-1} \beta$ is well defined. The above comments are still valid if $J$ and $K$ are exchanged. By the bijection $\gamma^{-1} \beta$, the following lemma is equivalent to Lemma $\mathrm{N}$.

Lemma $(\mathrm{P})$. For $m \geqslant 1$ we have

$$
\begin{aligned}
\nu\left(\left.P\right|_{m}, 0 / 1, J\right) & \equiv 1 \\
\nu\left(\left.P\right|_{2 m-1}, 1, K\right) & \equiv 1 \\
\nu\left(\left.P\right|_{2 m}, 0 / 2, K\right) & \equiv 1 .
\end{aligned}
$$

The third transformation $\delta$ is extended to the set of involutions $\sigma$ on $\left.P\right|_{m}$ such that all the transpositions are $J$-transpositions, by applying $\delta$ on every number in the cycle representation of $\sigma$. The transformation $\delta$ for involutions is reversible since $\delta$ for $N$ is reversible. For example

$$
\delta((15)(0,11)(12,7)(4)(16,3)(8))=(14)(1,10)(13,6)(5)(17,2)(9)
$$

If $(c, d)$ is a $J$-transposition then $(\delta(c), \delta(d))$ is still a $J$-transposition since $c+d=\delta(c)+$ $\delta(d)$.

The above comments are still valid if $J$ and $K$ are exchanged. Using the bijection $\delta$, we see that the following lemma is equivalent to Lemma $\mathrm{P}$.

Lemma (Q). For $m \geqslant 1$ we have

$$
\begin{aligned}
\nu\left(\left.Q\right|_{m}, 0 / 1, J\right) & \equiv 1 \\
\nu\left(\left.Q\right|_{2 m-1}, 1, K\right) & \equiv 1 \\
\nu\left(\left.Q\right|_{2 m}, 0 / 2, K\right) & \equiv 1 .
\end{aligned}
$$

Since Lemmas $N, P$ and $Q$ are equivalent, we prove (P1), (N2), (N3) in Section 3.

\section{The proofs}

We begin with several comments. The proofs of all the theorems and lemmas are based on induction on the lengths of the permutations. Small values of $m$ can be easily checked by hand. The proof of $(N 2)$ uses $(P 1)$, the proof of $(P 1)$ uses $(J 2)$, and the proof of $(J 2)$ uses again (N2). This is not a circular reasoning, because the length of permutations is smaller than the length of the original permutations. For every permutation $\sigma$, we say that $\sigma$ contains a column ( $\left.\begin{array}{c}\text { odd } \\ \text { even }\end{array}\right)$ (in the two-line representation) if there is some odd number $j$

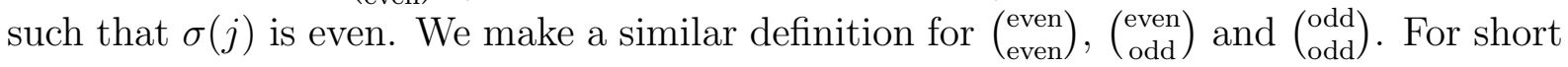


we say that a permutation $\sigma$ is "in (J1)" if $\sigma$ satisfies the conditions described in the statement of Theorem (J1), and that an involution $\sigma$ is "in (P1)" if $\sigma$ is an involution on the set $\left.P\right|_{m}$ having 0 or 1 fixed point, and all transpositions are $J$-transpositions. The following basic facts are easy to verify:

(FJ1) The set $J$ contains all even numbers;

(FJ2) If an odd number $x$ is in $J$, then $x \equiv 3(\bmod 4)$.

Proof of (J1). We count the permutations in (J1) modulo 2. If $\sigma$ contains more than two columns ( $\left.\begin{array}{l}\text { odd } \\ \text { odd }\end{array}\right)$ in the two-line representation, select the first two such columns $\left(\begin{array}{l}i_{1} \\ j_{1}\end{array}\right)$ and $\left(\begin{array}{l}i_{2} \\ j_{2}\end{array}\right)$. We define another permutation $\tau$ obtained from $\sigma$ by exchanging $j_{1}$ and $j_{2}$ in the bottom line. This procedure is reversible. By (FJ1), it is easy to verify that $\tau$ is also a valid permutation in (J1). So that we can delete the pair $\sigma$ and $\tau$, and there only remain the permutations containing 0 or 1 column ( $\left.\begin{array}{l}\text { odd } \\ \text { odd }\end{array}\right)$, in particular, having 0 or 1 odd fixed point. Similarly with an even number, there only remain permutations containing 0 or 1 column ( $\left.\begin{array}{c}\text { even } \\ \text { even }\end{array}\right)$. Consequently, the only remaining permutations have 0,1 or 2 fixed points. Thanks to the bijection $\sigma \mapsto \sigma^{-1}$, we need only consider the involutions. We can check that all transpositions are $J$-transpositions. If $m$ is odd, then the involution contains one fixed point, and the number of such involutions is $\nu\left(\left.N\right|_{m}, 1, J\right) \equiv 1$ by Lemma (N2). If $m$ is even, then the involution may contain 0 or 2 fixed points, and in the case of 2 fixed points, we can check that there are exactly one odd and one even fixed point. Hence the number of such involutions is $\nu\left(\left.N\right|_{m}, 0 / 2, J\right) \equiv 1$ by Lemma $(N 3)$.

Proof of (N2) and (N3). By (FJ2) the two numbers in every $J$-transposition are either both in $P$ or both in $Q$. This means that no $J$-transposition takes one number in $P$ and another in $Q$. The involutions in (N2) and (N3) are of type

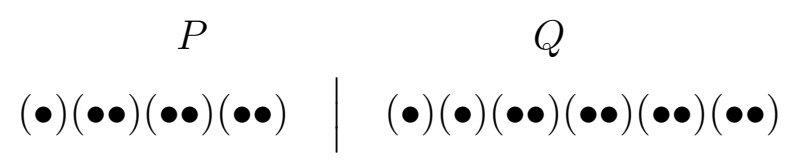

The two parts composed by numbers from $P$ and from $Q$ are "independent". The cardinalities of the two parts and the number of fixed points in each side are characterized by $m$. If $m=2 k+1$ is odd, then every involution $\sigma$ in (N2) has exactly one fixed point, in $\left.P\right|_{k}$ or $\left.Q\right|_{k+1}$, according to the parity of $k$. Hence

$$
\nu\left(\left.N\right|_{2 k+1}, 1, J\right) \equiv \nu\left(\left.P\right|_{k}, 0 / 1, J\right) \times \nu\left(\left.Q\right|_{k+1}, 0 / 1, J\right) \equiv 1 .
$$

The last equality follows from Lemmas $(P 1)$ and $(Q 1)$. If $m=2 k$ is even and $k$ is odd, then the cardinalities of $\left.P\right|_{k}$ and $\left.Q\right|_{k}$ are odd. So that there is one fixed point in $\left.P\right|_{k}$ and one in $\left.Q\right|_{k}$. Hence $\nu\left(\left.N\right|_{2 k}, 0, J\right)=0$ and

$$
\nu\left(\left.N\right|_{2 k}, 2, J\right) \equiv \nu\left(\left.P\right|_{k}, 1, J\right) \times \nu\left(\left.Q\right|_{k}, 1, J\right) \equiv 1 .
$$

Again, the last equality follows from Lemmas $(P 1)$ and $(Q 1)$. If $m=2 k$ is even and $k$ is even, then the cardinalities of $\left.P\right|_{k}$ and $\left.Q\right|_{k}$ are even. Three situations may occur: 
(i) no fixed point neither in $\left.P\right|_{k}$ nor in $\left.Q\right|_{k}$;

(ii) two fixed points in $\left.P\right|_{k}$ and no fixed point in $\left.Q\right|_{k}$;

(iii) two fixed points in $\left.Q\right|_{k}$ and no fixed point in $\left.P\right|_{k}$.

Hence, we get

$$
\begin{aligned}
\nu\left(\left.N\right|_{2 k}, 0, J\right) & \equiv \nu\left(\left.P\right|_{k}, 0, J\right) \times \nu\left(\left.Q\right|_{k}, 0, J\right) \equiv 1, \\
\nu\left(\left.N\right|_{2 k}, 2, J\right) & \equiv \nu\left(\left.P\right|_{k}, 0, J\right) \times \nu\left(\left.Q\right|_{k}, 2, J\right)+\nu\left(\left.P\right|_{k}, 2, J\right) \times \nu\left(\left.Q\right|_{k}, 0, J\right) \equiv 0 .
\end{aligned}
$$

The first equality follows from Lemmas $(P 1)$ and $(Q 1)$. The second equality is proved by using the bijection $\delta$ described in Section 2 .

Proof of (P1). When $m=2 k$ is even, the image $\tau=\gamma(\sigma)$ by $\gamma$ (described in Section 2) of every permutation $\sigma$ in $(P 1)$ can be identified with a permutation $\rho$ on $\{0,1, \ldots, k-1\}$ such that $i+\rho(i) \in J$ for $i=0,1, \ldots, k-1$. For example, taking $\sigma=(0,3)(7,8)(4,11)$, we have $\tau=\gamma(\sigma)=\gamma((0,3)(8,7)(4,11))=(0,0)(2,1)(1,2)$ which can be identified with the permutation on $\{0,1,2\}$ given by

$$
\rho=\left(\begin{array}{lll}
0 & 1 & 2 \\
0 & 2 & 1
\end{array}\right)
$$

The number of such permutations is odd by Theorem $(J 1)$.

When $m=2 k+1$ is odd, every permutation $\sigma$ in $(P 1)$ has one fixed point. Apply the transformation $\gamma$ to $\sigma$, then replace the unique singleton $b$ (which is obtained from the fixed point of $\sigma)$ by $(k, b)$ or $(b, k)$ to form a pair. There is a unique way to choose between $(k, b)$ and $(b, k)$ such that $\tau$ can be identified to a permutation $\rho$ on $\{0,1, \ldots, k\}$. For example take $\sigma=(0,11)(7,8)(3,12)(4)$, we have $\tau=\gamma(\sigma)=\gamma((0,11)(8,7)(12,3)(4))=$ $(0,2)(2,1)(3,0)(1)$, the single element is $b=1$, we need replace $(1)$ by $(1,3)$ (not $(3,1)$ of course). So that $\tau$ is identified to the following permutation on $\{0,1,2,3\}$

$$
\rho=\left(\begin{array}{cccc}
0 & 1 & 2 & 3 \\
2 & 3 & 1 & 0 \\
J & N & J & J
\end{array}\right)
$$

or its inverse

$$
\rho^{-1}=\left(\begin{array}{cccc}
0 & 1 & 2 & 3 \\
3 & 2 & 0 & 1 \\
J & J & J & N
\end{array}\right) .
$$

We can verify that one of the two permutations $\rho$ and $\rho^{-1}$ is in (J2) (look at the letter $N$ at the third line in the above examples). The number of such permutions is odd by Theorem (J2). 
Proof of (J2). For every permutation $\sigma$ in (J2), $i+\sigma(i) \in J$ for $i \leqslant m-2$. If $\sigma(m-1)$

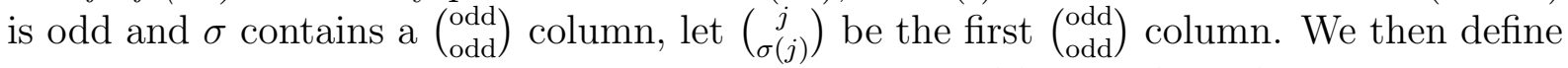
another permutation $\tau$ obtained from $\sigma$ by exchanging $\sigma(j)$ and $\sigma(m-1)$. This procedure is reversible. We can delete the pair $\sigma$ and $\tau$. Similarly we can delete every permutation $\sigma$ such that $\sigma(m-1)$ is even and $\sigma$ contains an $\left(\begin{array}{l}\text { even } \\ \text { even }\end{array}\right)$ column. There only remain two types of permutations. If $\sigma(m-1)$ is odd, then every number under an odd number in the two-line representation is even. If $\sigma(m-1)$ is even, then every number under an even number is odd, as shown here:

$$
\left(\begin{array}{cccccc|c}
1 & 2 & 3 & 4 & 5 & \cdots & m-1 \\
e & & e & & e & \cdots & o
\end{array}\right) \text { and }\left(\begin{array}{cccccc|c}
0 & 1 & 2 & 3 & 4 & \cdots & m-1 \\
o & & o & & o & \cdots & e
\end{array}\right)
$$

The letter "e" and "o" represent an even and odd numbers respectively. There are two cases to be considered.

(I) If $m$ is even, then $m-1$ is odd. The number of odd numbers in $\left.N\right|_{m}$ is equal to the number of even numbers in $\left.N\right|_{m}$.

(I.1) If $\sigma(m-1)$ is even, then the permutations are of the type

$$
\left(\begin{array}{lllllll|l}
0 & 1 & 2 & 3 & 4 & 5 & 6 & 7 \\
o & e & o & e & o & e & o & e \\
J & J & J & J & J & J & J & N
\end{array}\right)
$$

which is equivalent to the product

$$
\left(\begin{array}{llll}
0 & 2 & 4 & 6 \\
o & o & o & o \\
J & J & J & J
\end{array}\right) \times\left(\begin{array}{cccc}
1 & 3 & 5 & 7 \\
e & e & e & e \\
J & J & J & N
\end{array}\right)
$$

The first factor is equal to $\nu\left(\left.N\right|_{m}, 0, J\right)$, and the second factor is equivalent to

$$
\left(\begin{array}{llll}
1 & 3 & 5 \\
e & e & e & e \\
J & J & J &
\end{array}\right)
$$

which counts $\nu\left(\left.N\right|_{m-1}, 1, J\right) \equiv 1$ by $(\mathrm{N} 2)$.

(I.2) If $\sigma(m-1)$ is odd, then there are as many even numbers as odd numbers. Since the last column is ( $\left.\begin{array}{l}\text { odd } \\ \text { odd }\end{array}\right)$, there is necessarily a unique column ( $\left.\begin{array}{c}\text { even } \\ \text { even }\end{array}\right)$, which will be called "intruder", and marked by the $*$ sign. The permutations in $(J 2)$ are of type

$$
\sum_{* \text { even }}\left(\begin{array}{ccccccc|c}
0 & 1 & 2^{*} & 3 & 4 & 5 & 6 & 7 \\
o & e & e & e & o & e & o & o \\
J & J & J & J & J & J & J & N
\end{array}\right)
$$

The column 2 is the "intruder", and the letter $J$ under that column can be replaced by $N$. Exchanging $\sigma(*)$ and $\sigma(m-1)$ yields

$$
\sum_{* \text { even }}\left(\begin{array}{ccccccc|c}
0 & 1 & 2^{*} & 3 & 4 & 5 & 6 & 7 \\
o & e & o & e & o & e & o & e \\
J & J & N & J & J & J & J & N
\end{array}\right)
$$


which is equivalent to the product

$$
\left(\sum_{* \text { even }}\left(\begin{array}{cccc}
0 & 2^{*} & 4 & 6 \\
o & o & o & o \\
J & N & J & J
\end{array}\right)\right) \times\left(\begin{array}{cccc}
1 & 3 & 5 & 7 \\
e & e & e & e \\
J & J & J & N
\end{array}\right) .
$$

The first factor is equal to $\nu\left(\left.N\right|_{m}, 2, J\right)$ and the second one is equal to $\nu\left(\left.N\right|_{m-1}, 1, J\right) \equiv 1$ by (N2). By (I.1) and (I.2) the total number of permutations in this case is

$$
\nu\left(\left.N\right|_{m}, 0, J\right) \times \nu\left(\left.N\right|_{m-1}, 1, J\right)+\nu\left(\left.N\right|_{m}, 2, J\right) \times \nu\left(\left.N\right|_{m-1}, 1, J\right) \equiv \nu\left(\left.N\right|_{m}, 0 / 2, J\right) \equiv 1,
$$

where the last equality follows from (N3).

(II) If $m$ is odd, then $m-1$ is even. The number of even numbers in $\left.N\right|_{m}$ is equal to the number of odd numbers in $\left.N\right|_{m}$, plus 1 .

(II.1) If $\sigma(m-1)$ is even, then the permutations are of type

$$
\left(\begin{array}{llllllll|l}
0 & 1 & 2 & 3 & 4 & 5 & 6 & 7 & 8 \\
o & e & o & e & o & e & o & e & e \\
J & J & J & J & J & J & J & J & N
\end{array}\right)
$$

which is equivalent to the product

$$
\left(\begin{array}{llll}
0 & 2 & 4 & 6 \\
o & o & o & o \\
J & J & J & J
\end{array}\right) \times\left(\begin{array}{lllll}
1 & 3 & 5 & 7 & 8 \\
e & e & e & e & e \\
J & J & J & J & N
\end{array}\right) .
$$

The first factor is equal to $\nu\left(\left.N\right|_{m-1}, 0, J\right)$, and the second factor is equivalent to

$$
\left(\begin{array}{lllll}
1 & 3 & 5 & 7 \\
e & e & e & e & e \\
J & J & J & J &
\end{array}\right)
$$

The number of such permutations is equal to $\nu\left(\left.N\right|_{m}, 1, J\right)$, which is odd, by (N2).

(II.2) If $\sigma(m-1)$ is odd, the permutations are of type

$$
\sum_{* \text { even }}\left(\begin{array}{cccccccc|c}
0 & 1 & 2^{*} & 3 & 4 & 5 & 6 & 7 & 8 \\
o & e & e & e & o & e & o & e & o \\
J & J & J & J & J & J & J & J & N
\end{array}\right) .
$$

Exchanging $\sigma(*)$ and $\sigma(m-1)$ yields

$$
\sum_{* \text { even }}\left(\begin{array}{cccccccc|c}
0 & 1 & 2^{*} & 3 & 4 & 5 & 6 & 7 & 8 \\
o & e & o & e & o & e & o & e & e \\
J & J & N & J & J & J & J & J & N
\end{array}\right)
$$

which is equivalent to the product

$$
\left(\sum_{* \text { even }}\left(\begin{array}{cccc}
0 & 2^{*} & 4 & 6 \\
o & o & o & o \\
J & N & J & J
\end{array}\right)\right) \times\left(\begin{array}{ccccc}
1 & 3 & 5 & 7 & 8 \\
e & e & e & e & e \\
J & J & J & J & N
\end{array}\right) .
$$


The first factor counts the number of involutions of $\left.N\right|_{m-1}$ with 2 fixed points. Their number is equal to $\nu\left(\left.N\right|_{m-1}, 2, J\right)$. The second factor is equal to $\nu\left(\left.N\right|_{m}, 1, J\right) \equiv 1$ by (N2). By (II.1) and (II.2) the total number of permutations in this case is

$$
\nu\left(\left.N\right|_{m-1}, 0, J\right) \times \nu\left(\left.N\right|_{m}, 1, J\right)+\nu\left(\left.N\right|_{m-1}, 2, J\right) \times \nu\left(\left.N\right|_{m}, 1, J\right) \equiv \nu\left(\left.N\right|_{m-1}, 0 / 2, J\right) \equiv 1,
$$

where the last equality follows from (N3).

\section{Further results}

For subsets $A, B, C, D$ of $N$ let $\left|\begin{array}{ll}A & B \\ C & D\end{array}\right|_{m}$ denote the number of permutations $\sigma$ in $\mathfrak{S}_{m}$ such that

$$
i+\sigma(i) \in \begin{cases}A, & \text { if } i, \sigma(i) \in[0, m-2] \\ B, & \text { if } i \in[0, m-2], \sigma(i)=m-1 \\ C, & \text { if } i=m-1, \sigma(i) \in[0, m-2] \\ D, & \text { if } i=\sigma(i)=m-1 .\end{cases}
$$

Theorem (D). For every $m \geqslant 1$, we have

$$
\begin{aligned}
& \left|\begin{array}{ll}
J & J \\
J & J
\end{array}\right|_{m} \equiv 1, \\
& \left|\begin{array}{ll}
J & N \\
J & N
\end{array}\right|_{m} \equiv 1, \\
& \left|\begin{array}{ll}
J & N \\
N & N
\end{array}\right|_{m} \equiv m, \\
& \left|\begin{array}{ll}
K & K \\
K & K
\end{array}\right|_{m} \equiv m+1, \\
& \left|\begin{array}{ll}
K & N \\
K & K
\end{array}\right|_{m} \equiv m+1, \\
& \left|\begin{array}{ll}
K & N \\
N & K
\end{array}\right|_{m} \equiv m+1 .
\end{aligned}
$$

Remark 1. The following basic facts are easy to verify:

(FK1) The set $K$ contains no even integer;

(FK2) The set $K$ contains all integers of the form $4 n+1$;

Remark 2. Since $J$ contains all even numbers, taking $D=J$ is equivalent to taking $D=N$; Since $K$ does not contain any even number, taking $D=K$ is equivalent to taking $D=\emptyset$.

Remark 3. Formula (K1) implies that the Hankel determinants of the series

$$
\mathcal{K}(z)=\frac{1}{1-z}+\sum_{k \geqslant 0} \frac{z^{2^{n}-1}}{1-z^{2^{n}}}
$$


satisfies $H_{m}(\mathcal{K}) \equiv m+1$ for $m \geqslant 1$.

Remark 4. The formulas in Theorem $\mathrm{D}$ can be used to derive other formulas, for example

$$
\left|\begin{array}{cc}
J & K \\
K & J
\end{array}\right|_{m} \equiv m
$$

Proof of (Ex). We have

$$
\begin{aligned}
& \left|\begin{array}{cc}
J & N \\
N & \emptyset
\end{array}\right|_{m} \equiv\left|\begin{array}{cc}
J & N \\
N & N
\end{array}\right|_{m}-\left|\begin{array}{ll}
J & J \\
J & J
\end{array}\right|_{m-1} \equiv m-1 \quad[\operatorname{By}(J 3) \text { and }(J 1)] \text {, } \\
& \left|\begin{array}{ll}
J & N \\
J & \emptyset
\end{array}\right|_{m} \equiv\left|\begin{array}{ll}
J & N \\
J & N
\end{array}\right|_{m}-\left|\begin{array}{ll}
J & J \\
J & J
\end{array}\right|_{m-1} \equiv 1-1 \equiv 0, \\
& \left|\begin{array}{ll}
J & J \\
J & \emptyset
\end{array}\right|_{m} \equiv\left|\begin{array}{cc}
J & J \\
J & N
\end{array}\right|_{m}-\left|\begin{array}{ll}
J & J \\
J & J
\end{array}\right|_{m-1} \equiv 1-1 \equiv 0, \\
& \left|\begin{array}{cc}
J & N \\
K & \emptyset
\end{array}\right|_{m} \equiv\left|\begin{array}{cc}
J & N \\
N & \emptyset
\end{array}\right|_{m}-\left|\begin{array}{cc}
J & N \\
J & \emptyset
\end{array}\right|_{m} \equiv(m-1)-0 \equiv m-1, \\
& \left|\begin{array}{ll}
J & J \\
K & \emptyset
\end{array}\right|_{m} \equiv\left|\begin{array}{cc}
J & J \\
N & \emptyset
\end{array}\right|_{m}-\left|\begin{array}{ll}
J & J \\
J & \emptyset
\end{array}\right|_{m} \equiv 0-0 \equiv 0, \\
& \left|\begin{array}{cc}
J & K \\
K & \emptyset
\end{array}\right|_{m} \equiv\left|\begin{array}{cc}
J & N \\
K & \emptyset
\end{array}\right|_{m}-\left|\begin{array}{cc}
J & J \\
K & \emptyset
\end{array}\right|_{m} \equiv(m-1)-0 \equiv m-1, \\
& \left|\begin{array}{cc}
J & K \\
K & J
\end{array}\right|_{m} \equiv\left|\begin{array}{cc}
J & K \\
K & \emptyset
\end{array}\right|_{m}+\left|\begin{array}{ll}
J & J \\
J & J
\end{array}\right|_{m-1} \equiv(m-1)+1 \equiv m \text {. }
\end{aligned}
$$

The first two identities $(J 1)$ and $(J 2)$ are simply reproduced from Theorem J.

Proof of (J3). By the same arguments as in the proof of (J1), the permutations to enumerate are the involutions $\sigma$ such that

(i) $\sigma$ has $0,1,2$ fixed points;

(ii) every transposition in $\sigma$ is a $J$-transposition, except the one which contains $m-1$, if it exists;

(iii) If $m-1$ is in a transposition $(m-1, b)$, then $m-1+b$ is odd.

(J3.1) If $m$ is odd, then there is exactly one fixed point. If $m-1$ is this fixed point, then the number of involutions is $\nu\left(\left.N\right|_{m-1}, 0, J\right)$. If $m-1$ is in a transposition $\left(b_{1}, m-1\right)$ and the fixed point is $\left(b_{2}\right)$, then deleting $m-1$ is a transformation reversible. We get an involution of $\mathfrak{S}_{m-1}$ with two fixed points $b_{1}, b_{2}$. The number of such involutions is $\nu\left(\left.N\right|_{m-1}, 2, J\right)$. Hence the total number of involutions is $\nu\left(\left.N\right|_{m-1}, 0, J\right)+\nu\left(\left.N\right|_{m-1}, 2, J\right)=$ $\nu\left(\left.N\right|_{m-1}, 0 / 2, J\right)$, which is an odd number by Theorem (N3).

(J3.2) If $m$ is even, three situations have to be considered.

(i) If the involution has no fixed point, then the number of involutions is $\nu\left(\left.N\right|_{m-1}, 1,0\right)$. 
(ii) If the involution $\sigma$ has 2 fixed points and $m-1$ is a fixed point, then the number of involutions is also $\nu\left(\left.N\right|_{m-1}, 1,0\right)$.

(iii) If $m-1$ is in a transposition $\left(m-1, b_{1}\right)$ and the two fixed points are $\left(b_{2}\right)$ and $\left(b_{3}\right)$, then $b_{2}+b_{3}$ is odd. Exchanging $b_{1}$ with $b_{2}\left(\right.$ resp. $\left.b_{3}\right)$ if $b_{2} \equiv b_{1}(\bmod 2)\left(\operatorname{resp} . b_{3} \equiv b_{1}\right.$ $(\bmod 2))$ is a transformation reversible. Hence the total number of involutions is equal to $\nu\left(\left.N\right|_{m-1}, 1,0\right)+\nu\left(\left.N\right|_{m-1}, 1,0\right)$ plus an even number, which is 0 modulo 2 .

Proof of (K1). By (FK1) every permutation in $(K 1)$ contains no fixed point. Since we need only count the involutions, the number of such permutations is 0 if $m$ is odd. If $m=2 k$ is even, the permutations are of type

$$
\left(\begin{array}{cccccccc}
0 & 1 & 2 & 3 & 4 & 5 & 6 & 7 \\
o & e & o & e & o & e & o & e \\
K & K & K & K & K & K & K & K
\end{array}\right)
$$

which is equivalent to the product

$$
\left(\begin{array}{cccc}
0 & 2 & 4 & 6 \\
o & o & o & o \\
K & K & K & K
\end{array}\right) \times\left(\begin{array}{cccc}
1 & 3 & 5 & 7 \\
e & e & e & e \\
K & K & K & K
\end{array}\right) .
$$

The two factors are equal to $\nu\left(\left.N\right|_{m}, 0, K\right) \equiv 1$ by (N1).

Proof of (K2). If $m$ is even, all permutations $\sigma$ in $\left|\begin{array}{ll}K & N \\ K & N\end{array}\right|$ are of type

$$
\left(\begin{array}{cccccccc}
0 & 1 & 2 & 3 & 4 & 5 & 6 & 7 \\
o & e & o & e & o & e & o & e \\
K & K & K & K & K & K & K & N
\end{array}\right)
$$

which is equivalent to the product

$$
\left(\begin{array}{cccc}
0 & 2 & 4 & 6 \\
o & o & o & o \\
K & K & K & K
\end{array}\right) \times\left(\begin{array}{cccc}
1 & 3 & 5 & 7 \\
e & e & e & e \\
K & K & K & N
\end{array}\right)
$$

The number of permutations of the type given by the first factor is equal to $\nu\left(\left.N\right|_{m}, 0, K\right) \equiv$ 1 and that of the type given by the second one is equal to $\nu\left(\left.N\right|_{m-1}, 1, K\right) \equiv 1$ by $(\mathrm{N} 1)$.

If $m$ is odd, all permutations $\sigma$ in $\left|\begin{array}{ll}K & N \\ K & N\end{array}\right|_{m}$ are of type

$$
\left(\begin{array}{ccccccccc}
0 & 1 & 2 & 3 & 4 & 5 & 6 & 7 & 8 \\
o & e & o & e & o & e & o & e & e \\
K & K & K & K & K & K & K & K & N
\end{array}\right)
$$


which is equivalent to the product

$$
\left(\begin{array}{cccc}
0 & 2 & 4 & 6 \\
o & o & o & o \\
K & K & K & K
\end{array}\right) \times\left(\begin{array}{ccccc}
1 & 3 & 5 & 7 & 8 \\
e & e & e & e & e \\
K & K & K & K & N
\end{array}\right)
$$

The number of permutations of the type given by the first factor is equal to $\nu\left(\left.N\right|_{m-1}, 0, K\right)$ $\equiv 1$ and that of the type given by the second one is equal to $\nu\left(\left.N\right|_{m}, 1, K\right) \equiv 1$ by (N1). Hence

$$
\left|\begin{array}{ll}
K & N \\
K & N
\end{array}\right|_{m} \equiv 1
$$

and

$$
\left|\begin{array}{ll}
K & N \\
K & K
\end{array}\right|_{m} \equiv\left|\begin{array}{ll}
K & N \\
K & N
\end{array}\right|_{m}-\left|\begin{array}{ll}
K & K \\
K & K
\end{array}\right|_{m-1} \equiv 1-(m-1+1) \equiv m+1
$$

Proof of (K3). We need only count the involutions without fixed points, so that the number of such permutations is 0 when $m$ is odd. If $m=2 k$ is even, all transpositions are necessarily $K$-transpositions, except the one which contains $m-1$. Removing $m-1$ yields an involution with one fixed point. The number of such involutions is $\nu\left(\left.N\right|_{m-1}, 1, K\right) \equiv 1$ by (N1).

Furthermore, in Lemma (N3) we have a mixed formula for 0 or 2 fixed points. From the proof of (N2) and (N3) we can separate it into two more precise formulas.

Lemma (N'). We have

$$
\begin{aligned}
& \nu\left(\left.N\right|_{2 k}, 0, J\right) \equiv k+1, \\
& \nu\left(\left.N\right|_{2 k}, 2, J\right) \equiv k .
\end{aligned}
$$

Using the transfomations $\beta, \gamma, \delta$, we see that Lemma $\mathrm{N}^{\prime}$ is equivalent to

Lemma $\left(\mathrm{P}^{\prime}\right)$. We have

$$
\begin{aligned}
& \nu\left(\left.P\right|_{2 k}, 0, K\right) \equiv k+1, \\
& \nu\left(\left.P\right|_{2 k}, 2, K\right) \equiv k .
\end{aligned}
$$

Lemma (Q'). We have

$$
\begin{aligned}
& \nu\left(\left.Q\right|_{2 k}, 0, K\right) \equiv k+1, \\
& \nu\left(\left.Q\right|_{2 k}, 2, K\right) \equiv k .
\end{aligned}
$$




\section{Completion of the combinatorial proofs of Theo- rems $A P W W$ and $C$}

Recall that

$$
J=\left\{(2 n+1) 2^{2 k}-1 \mid n, k \in N\right\}=\{0,2,3,4,6,8,10,11, \ldots\}
$$

and

$$
K=N \backslash J=\{1,5,7,9,13,17, \ldots\}=\left\{(2 n+1) 2^{2 k+1}-1 \mid n, k \in N\right\} .
$$

First, we notice that, for $k \geqslant 1$, the integer $g_{k}$ is equal to the 2 -adic valuation of $2 k$, known sometimes as the ruler function. For $k \geqslant 1$, we have the recursion

$$
g_{2 k+1}=1 \quad \text { and } \quad g_{2 k}=1+g_{k} .
$$

This sequence starts as

$$
\left(g_{k}\right)_{k \geqslant 1}=1,2,1,3,1,2,1,4,1,2,1,3,1,2,1,5,1,2,1,3,1,2,1,4, \ldots
$$

The proofs of Theorems APWW and C combine Theorem J with the next two lemmas.

Lemma 1. For $k \geqslant 0$, the integer $g_{k+1}$ is odd if, and only if, $k$ is in $J$.

Proof. Let $k$ be a non-negative integer. It follows from (1.1) that $g_{k+1}$ is equal to the number of pairs $(j, m)$ of integers $j \geqslant 1, m \geqslant 0$ such that $j 2^{m}=k+1$. In particular, writing $k+1=j_{0} 2^{m_{0}}$ with $j_{0}$ odd, we see that

$$
k+1=j_{0} 2^{m_{0}}=\left(2 j_{0}\right) 2^{m_{0}-1}=\ldots=\left(2^{m_{0}} j_{0}\right) 2^{0},
$$

showing that $g_{k+1}=m_{0}+1$. Consequently, $g_{k+1}$ is odd if, and only if, $m_{0}$ is even, that is, if and only if, $k$ is in $J$.

Lemma 2. For $k \geqslant 0$, the integer $\delta_{k}:=\left(t_{k+1}-t_{k}\right) / 2$ is odd if, and only if, $k$ is in $J$.

Proof. For $\ell \geqslant 0$, we have

$$
\delta_{2 \ell}=\left(t_{2 \ell+1}-t_{2 \ell}\right) / 2=-t_{\ell}= \pm 1,
$$

thus $\delta_{2 \ell}$ is odd. For an odd integer $j \geqslant 1$ and an integer $k \geqslant 1$, we have

$$
\delta_{j 2^{2 k}-1} \equiv\left(t_{j 2^{2 k}}-t_{j 2^{2 k}-1}\right) / 2 \equiv\left(t_{j 2^{2 k-1}}+t_{j 2^{2 k-1}-1}\right) / 2 \equiv 1+\delta_{j 2^{2 k-1}-1} \quad(\bmod 2)
$$

and, likewise,

$$
\delta_{j 2^{2 k-1}-1} \equiv 1+\delta_{j 2^{2(k-1)}-1}(\bmod 2) .
$$

Consequently, an immediate induction shows that, for any odd integer $j$ and any integer $\ell \geqslant 0$, the integer $\delta_{j 2^{\ell}-1}$ is odd if, and only if, $\ell$ is even, that is, if, and only if, $j 2^{\ell}-1$ is in $J$. This proves the lemma. 
Proof of Theorem $C$. Let $\mathcal{J}(z)$ be the power series

$$
\mathcal{J}(z)=\sum_{k \geqslant 0} j_{k} z^{k}=1+z^{2}+z^{3}+z^{4}+z^{6}+\ldots
$$

defined by $j_{k}=1$ if $k \in J$ and $j_{k}=0$ otherwise. Let $k$ be a positive integer. By the definition of the determinant, $H_{k}(\mathcal{J})$ is equal to

$$
\sum_{\sigma \in \mathfrak{S}_{k}}(-1)^{i n v(\sigma)} j_{0+\sigma(0)} j_{1+\sigma(1)} \cdots j_{k-1+\sigma(k-1)},
$$

where $i n v(\sigma)$ is the number of inversions of $\sigma$. The product $j_{0+\sigma(0)} j_{1+\sigma(1)} \cdots j_{k-1+\sigma(k-1)}$ is equal to 1 if $i+\sigma(i) \in J$ for $i=0,1, \ldots, k-1$, and is equal to 0 otherwise. Hence, the above Hankel determinant $H_{k}(\mathcal{J})$ is equal modulo 2 to the number of permutations $\sigma \in \mathfrak{S}_{k}$ such that $i+\sigma(i) \in J$ for all $i=0,1, \ldots, k-1$, which is equal to 1 modulo 2 by Theorem $(\mathrm{J} 1)$. We deduce from Lemma 1 that $H_{k}(\mathcal{J}) \equiv H_{k}^{1}(\mathcal{G})$. Consequently, the Hankel determinant $H_{k}^{1}(\mathcal{G})$ is always an odd integer. Furthermore, it follows from (1.1) that the coefficients of the power series $\mathcal{F}(z)$ and $\mathcal{G}(z)$ are congruent modulo 2. This implies that $H_{k}^{1}(\mathcal{F}) \equiv H_{k}^{1}(\mathcal{G})$, proving that the Hankel determinant $H_{k}^{1}(\mathcal{F})$ is always an odd integer.

Proof of Theorem $A P W W$. Let $k$ be a positive integer and consider the Hankel determinant $H_{k}(\mathcal{T})$ of the matrix

$$
\left(\begin{array}{cccc}
t_{0} & t_{1} & \ldots & t_{k-1} \\
t_{1} & t_{2} & \ldots & t_{k} \\
\vdots & \vdots & \ddots & \vdots \\
t_{k-1} & t_{k} & \ldots & t_{2 k-2}
\end{array}\right)
$$

For $j=1, \ldots, k-1$, subtracting the $(j+1)$-th column from the $j$-th column, we see that $H_{k}(\mathcal{T})$ is equal to the determinant of the matrix

$$
\left(\begin{array}{ccccc}
t_{0}-t_{1} & t_{1}-t_{2} & \ldots & t_{k-2}-t_{k-1} & t_{k-1} \\
t_{1}-t_{2} & t_{2}-t_{3} & \ldots & t_{k-1}-t_{k} & t_{k} \\
\vdots & \vdots & \ddots & \vdots & \vdots \\
t_{k-1}-t_{k} & t_{k}-t_{k+1} & \ldots & t_{2 k-3}-t_{2 k-2} & t_{2 k-2}
\end{array}\right) .
$$

By Lemma 2, this determinant is equal to $2^{k-1}$ times an integer which has the same parity as the determinant of the matrix

$$
\left(\begin{array}{ccccc}
j_{0} & j_{1} & \cdots & j_{k-2} & t_{k-1} \\
j_{1} & j_{2} & \cdots & j_{k-1} & t_{k} \\
\vdots & \vdots & \ddots & \vdots & \vdots \\
j_{k-1} & j_{k} & \cdots & j_{2 k-3} & t_{2 k-2}
\end{array}\right)
$$

where $j_{0}, j_{1}, \ldots$ are defined in the proof of Theorem C. Now, we apply Theorem (J2) to deduce that the determinant of the latter matrix is an odd integer. It then follows that the Hankel determinant $H_{k}(\mathcal{T})$ is a nonzero integer. 


\section{References}

[1] J.-P. Allouche, J. Peyrière, Z.-X. Wen, and Z.-Y. Wen. Hankel determinants of the Thue-Morse sequence. Ann. Inst. Fourier (Grenoble), 48:1-27, 1998.

[2] Y. Bugeaud. On the irrationality exponent of the Thue-Morse-Mahler numbers. Ann. Institut Fourier (Grenoble), 61:2065-2076, 2011.

[3] M. Coons. On the rational approximation of the sum of the reciprocals of the Fermat numbers. The Ramanujan Journal, 30:39-65, 2013.

[4] K. Mahler. Arithmetische Eigenschaften der Lösungen einer Klasse von Funktionalgleichungen. Math. Ann., 101:342-366, 1929. Corrigendum 103:532, 1930.

[5] W. M. Schmidt. Diophantine Approximation. Lecture Notes in Math., 785, Springer, Berlin, 1980. 